\title{
Tricuriasis: Causa de diarrea crónica y sangrado digestivo
}

\section{Trichuriasis: Cause of diarrhea and gastrointestinal Bleeding}

\author{
Misael Alonso Pineda Santos* Luis Enrique Jovel Banegas**
}

\section{RESUMEN}

La tricuriasis es una enfermedad que se estima que afecta a 800 millones de personas y que su mayor prevalencia ocurre entre las personas de 5 a 15 años. La mayoría de las infecciones son asintomáticas, pero las infecciones masivas pueden causar síntomas gastrointestinales. Como los demás helmintos transmitidos por la tierra, el trichuris se distribuye globalmente en el trópico y sub trópico y es muy común en personas con nivel socioeconómico bajo.

En Honduras, país en vías de desarrollo, se pueden observar casos en los que la tricuriasis puede llegar a dar sus complicaciones más graves, como ser sangrado digestivo, diarrea prolongada y prolapso rectal.

\section{PALABRAS CLAVE}

Diarrea, prolapso rectal, sangrado digestivo, Tricuriasis.

\section{ABSTRACT}

Trichuriasis is a disease that affects 800 million people. The highest prevalence occurs among people 5-10 years old. Most infections are asymptomatic, but massive infections can cause gastrointestinal symptoms. Like other soil-transmitted helminths, the Trichuris is globally distributed in the tropics and subtropics and is very common in people with low socioeconomic status.

In Honduras, a developing country, there are cases where the Trichuris can cause its most serious complications, such as gastrointestinal bleeding and prolonged diarrhea and rectal prolapse.

*Médico Residente de segundo año de Pediatría, Universidad Nacional Autónoma de Honduras en el Valle de Sula UNAH-VS

** Gastroenterólogo Pediatra HNMCR

Dirigir correspondencia: misaelpineda@gmail.com

Recibido: 11/Jun/2015, aprobado:22/Jul/2015

\section{KEYWORD}

Diarrhea, rectal prolapse, Trichuriasis.

\section{INTRODUCCIÓN}

Tricuriasis es causada por la infección con Trichuris trichiura, nematodo que vive enhebrado en las paredes del colon, principalmente en el ciego, y en recto en infecciones intensas. ${ }^{(1)}$ Las infecciones crónicas e intensas, determinadas por una cuenta de huevos, de 20 o más en $2 \mathrm{mg}$ de heces, están asociadas a cuadros disentéricos serios, anemia, desnutrición y falla de medro. Puede haber prolapso rectal. ${ }^{(2,3)}$

A continuación se reporta un caso de tricuriasis atendido en la sala de Medicina Pediátrica del Hospital Mario Catarino Rivas y se realiza una revisión de la patología, su evolución clínica, abordaje y tratamiento.

\section{CASO CLÍNICO}

Paciente masculino de 8 años de edad, procedente de Yoro, Yoro, es referido a la emergencia del Hospital Mario Catarino Rivas por presentar diarrea, sangrado digestivo bajo de 6 meses de evolución.

Paciente fue manejado en el centro de salud y Hospital de Yoro, empíricamente como una parasitosis, sin realizar exámenes de laboratorio, dando piperazina y albenzadol. Al no haber mejoría y ya que el paciente había presentado en esa semana 2 veces prolapso rectal, fue referido a nuestro hospital.

A su ingreso el paciente se encontraba asintomático, pero al examen físico se observaba con palidez generalizada y se notaba que su estatura era baja para su edad, se ingresa como una diarrea crónica y sangrado digestivo bajo en estudio. 
Durante su estudio se obtuvieron los siguientes resultados: el hemograma con Hemoglobina de $7.2 \mathrm{~g} / \mathrm{dl}$, los glóbulos blancos con 5,600 (neutrófilos del $40.6 \%$, Linfocitos del $45.3 \%$ ), las plaquetas de 316,000, El examen General de Heces reportó Wright Negativo, se observaron más de 20 huevos de Trichuris trichiuria en $2 \mathrm{gr}$ de heces, y el guayaco: positivo.

Fue evaluado por el servicio de Gastroenterología Pediátrica quien realizó una colonoscopia para investigar otra causa del sangrado digestivo en este paciente, previa transfusión de hemoderivados por la anemia.

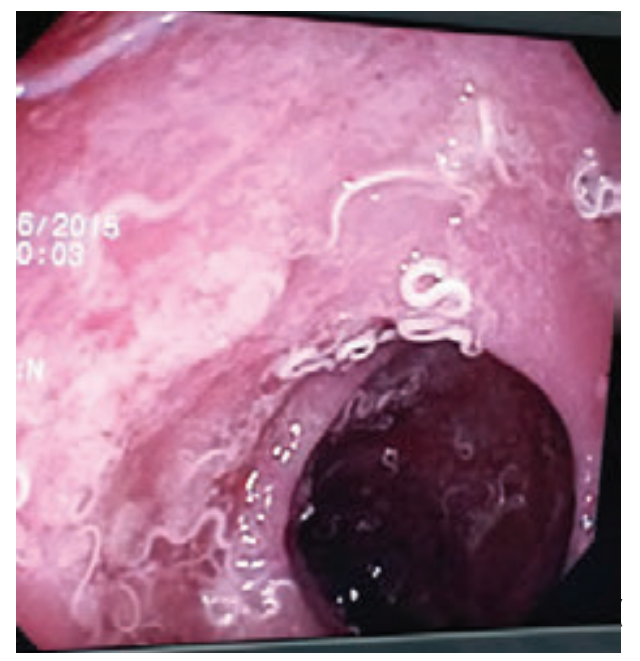

Figura No. 1: Imagen de colonoscopía que muestra el colon sigmoides cubierto de Trichuris trichiuria.

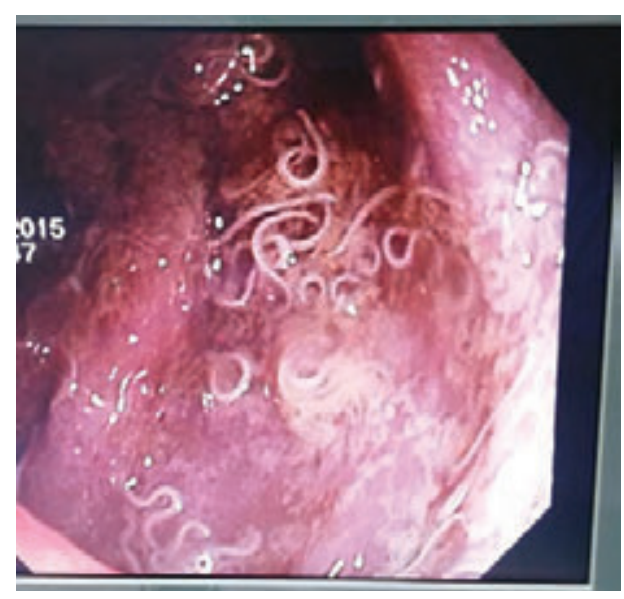

Figura No. 2: Imagen de colonoscopía que muestra el colon transverso cubierto por Trichuris trichiuria.
El estudio de colonoscopia descarta alguna otra patología causante de la diarrea y el sangrado de este paciente y confirma la presencia de una parasitosis masiva en todo el trayecto del intestino que se extendía desde colon ascendente hasta ano.

Se inició tratamiento con mebendazol $100 \mathrm{mg}$ vía oral BID por 3 días a repetir misma dosis en dos semanas, con hierro suplementario.

\section{DISCUSION:}

Por lo expuesto en este caso, el paciente se encuentra dentro de los rangos de edad más frecuentes en los que afecta la tricuriasis. Clínicamente se veía afectado con las manifestaciones clínicas graves que puede dar esta parasitosis y las repercusiones a largo plazo solo se podrán ver con un seguimiento periódico del paciente, ya que la anemia se puede corregir con suplementos de hierro, el sangrado digestivo y la diarrea con el tratamiento adecuado para la tricuriasis, pero la afectación en su estatura y saber si puede haber algún tipo de trastorno a nivel cognitivo o intelectual pueden ser irreversibles si no se siguen las adecuadas medidas de soporte nutricional y medidas de higiene personal y familiar.

La tricuriasis afecta más a personas entre los 5-15 años de edad, en Honduras en el Hospital Escuela se reconoce un $6 \%$ de infección (todas las edades) y alrededor de 30 infecciones severas anuales que requieren hospitalización. ${ }^{(4)} \mathrm{En}$ su mayoría, las personas infectadas son de procedencia rural con un nivel socioeconómico pobre.

Las hembras (35-50 mm de largo) y los machos (30-40 $\mathrm{mm}$ de largo) adultos viven enhebrados en el lumen intestinal. Las hembras depositan huevos de cascara gruesa y de forma ovoide, con tapones mucoides en los extremos, que son expulsados en las heces y contaminan el suelo por deposición al aire libre. En el suelo tardan unas 3 semanas en desarrollarse a su estado infectivo. Al ingerir los huevos se liberan larvas que penetran la mucosa del intestino 
delgado y después de 4 mudas emergen juveniles inmaduros que pasivamente pasan al colon. ${ }^{(5,6)}$

Las infecciones leves no tienen significado clínico. Infecciones con 30 huevos o más en 2 mg de heces pueden ser clínicamente significativas. ${ }^{(1,3)}$ La tricuriasis crónica, se acompaña de evacuaciones con moco y sangre, dolor abdominal, pérdida de apetito, edema facial y pedio, desnutrición, anemia o prolapso rectal. Como secuela se puede presentar como retraso en el crecimiento y déficit en el desarrollo cognitivo e intelectual. La anemia se debe a hemorragia por la colitis disentérica y prolapso rectal. Cada gusano succiona $0.005 \mathrm{ml}$ sangre/ d. ${ }^{(7,8,9)}$

En pacientes con sospecha clínica y epidemiológica, como ser niños o adultos jóvenes con anemia, disentería crónica, edema y retraso de crecimiento, hay que solicitar examen general de heces y exigir una cuenta de huevos, un examen hematológico para determinar hemoglobina y eosinofilia. ${ }^{(1)}$ Cuando el resultado del examen de heces es negativo, se puede realizar una colonoscopia buscando T. trichiura enhebrados en la mucosa inflamada y edematosa. ${ }^{(3)} \mathrm{Si}$ esto se comprueba, se procede a tratamiento específico y sintomático.

El tratamiento de elección es el mebendazol 100mg, dos veces al día, por tres días o 500mg dosis única, es el tratamiento para todas las edades. ${ }^{(3,10)}$ El albendazol a dosis de $400 \mathrm{mg}$ v.o. por 3 días o ivermectina $200 \mu \mathrm{g} / \mathrm{kg} /$ día v.o. por 3 días son tratamientos alternativos.

El prolapso se corrige al abatir la carga parasitaria y mejorar el estado nutricional del paciente, puede ser reducido manualmente o requerir consultar un proctólogo. ${ }^{(10)}$

Es necesario verificar si hay expulsión de gusanos por lavado de heces o ausencia de huevos por examen directo de heces dos semanas después del tratamiento. Si persiste la infección, demostrada por la presencia de huevos en el examen de heces, repetir dosis de medicamento. ${ }^{(3)}$

Se debe corregir la anemia y administrar micronutrientes. Reforzar las medidas de higiene personal y pública, consiste en hacer la correcta eliminación de las excretas, dotar de agua potable a las comunidades, hervir el agua de consumo, lavar las frutas y verduras antes de comerlas. $^{(5)}$

Con tratamiento adecuado y sin reinfecciones el pronóstico es excelente. Si el daño ha sido severo y prolongado, como por ejemplo falla del crecimiento, es posible que este no se revierta totalmente.

\section{BIBLIOGRAFÍA}

1. Bravo T. Trichuriasis: Epidemiología, diagnóstico y tratamiento. Rev Mex Pediatr [internet] 2004 [citado 10 Jun 2015]; 71(6); 299-305. Disponible en: http://www.medi graphic.com/pdfs/pediat/sp-2004/sp046j. pdf.

2. Lawrence M,Tienney Jr, McPhee S. Diagnóstico Clínico y Tratamiento. México: Editorial El Manual Moderno; 2000.

3. Instituto de Enfermedades Infecciosas y
Parasitología Antonio Vidal. Manual de Manejo de Enfermedades Parasitarias Prioritarias en Honduras. 2a. Edición. Tegucigalpa, Honduras: Organización Panamericana de la Salud / [AZER Impresos]; 2009.

4. Kaminsky RG, Retes E. Helmintiasis en niños en Amapala, Honduras. Honduras Pediatrica [internet] 2000 [citado 10 Jun 2015]. 21(2): Abril, May, Junio. Disponible en: http://cidbimena.desastres.hn/RHP/pdf /2000/pdf/Vol21-2-2000-4.pdf. 
5. Kaminsky RG., Javier A., Castillo V. Prevalencia de Helmintos en niños. Municipio de Santa Ana, Honduras. Honduras Pediatrica [internet] 2000 [citado 10 Jun 2015]. 21(2): Abril, May, Junio. Disponible en: http://cid bimena.desastres.hn/RHP/pdf/2000/pdf/ Vol21-2-2000-3.pdf.

6. Acha PN, Szyfres B (Pan American Health Organization [PAHO]). Zoonoses and communicable diseases common to man and animals. Volume 3. Parasitoses. 3rd ed. Washington DC: PAHO; 2003. Scientific and Technical Publication No. 580. Trichuriasis of animal origin; p. 302-305.

7. Iwashita AT. Parasitosis. Tópicos selectos en medicina interna - Gastroenterologia. 1 ed. Peru: Impresiones Santa Ana SAC; 2006. p. 281-294.
8. Martínez A. Sierra C. Parasitosis intestinales. En: Cruz M, Manual de Pediatría. 2a edición. Madrid:Ergon; 2008. p. 659-665.

9. Werner A. Infecciones por parásitos más frecuentes y su manejo. Rev Med Clin CONDES [internet] 2014 [citado 10 Jun 2015]; 25(3):485-528. Disponible en: http://www.clinicalascondes.cl/Dev_CLC/media/Imagenes/PDF $\% 20$ revista\% 20m\%C3\%A9dica/2014/3\%20abril/12-Dr. Apt.pdf.

10. Carlos S. Manual de Pediatría ambulatoria. Parte cap. 40 Parasitología. Santiago, Chile: Editorial Mediterraneo; 2013. Páginas 501511. 